\title{
PROTEOMICS IN OBESITY RESEARCH
}

\author{
Sílvia Barceló-Batllori \\ Proteomics Unit, Aragon Health Sciences Institute (ICS), Zaragoza, Spain
}

Obesity has emerged as one of the major global epidemics of the 21 st century and is now reaching alarming proportions. Obese subjects have an increased morbidity and mortality, decreased quality of life, and a major risk of developing co-morbidities such as diabetes and insulin resistance. Obesity is a complex disease characterised by an increase in body fat mass resulting from an imbalance between energy intake and expenditure. Proteomics may be useful in unravelling the pathogenesis of obesity, since a combination of genetic predisposition and environmental factors account for its development.

Adipose tissue plays a major role in obesity, not only for its capacity to store and metabolise lipids, but also for its ability to act as an endocrine organ. Adipose tissue is a complex organ and contains several cell types, namely the adipocytes, and stromal fraction (macrophages, endothelial cells, vascular cells). We are currently investigating the alterations in the adipocyte and stromal fraction proteome of visceral adipose tissue. Tissue samples were obtained from obese subjects (body mass index, BMI $>40 \mathrm{~kg} /$ $\mathrm{m}^{2}$ ) undergoing bariatric surgery with different degrees of glucose tolerance (diabetic, glucose intolerant and normal) and lean subjects (BMI $20-25 \mathrm{~kg} / \mathrm{m}^{2}$ ). Protein extracts were analysed by DIGE, and proteins identified by MS/MS. Candidate proteins in the adipocyte fraction overexpressed in obesity include carboxylesterase, GRP78, transketolase, catalase; and hsp60, UQCRC1 and perilipin were down-regulated.

Target identification of the potential anti-obesity agent sodium tungstate has also been investigated using 2D and DIGE. Oral administration of tungstate reduced body weight gain and adiposity, and improved dyslipemia and insulin resistance in cafeteria dietinduced rats. Tungstate is currently undergoing phase II clinical trials. In white adipose tissue we found that tungstate treatment reversed the expression changes of $70 \%$ of the proteins modified in obesity and was able to modulate cellular structure, metabolism, redox, and signalling processes. In brown adipose tissue, tungstate modulates redox processes and increases energy dissipation through uncoupling and up-regulation of PGC-1 $\alpha$ (peroxisome proliferator-activated receptor $\gamma$ coactivator $1 \alpha$ ).

The work presented here was mainly done at the Laboratory of Diabetes and Obesity, IDIBAPS, Hospital Clínic de Barcelona, Catalonia, Spain. 